\title{
APPLICATION OF FUZZY LOGIC REASONING MODEL FOR DETERMINING ADHESIVE STRENGTH OF THIN FLIM COATINGS
}

\author{
Ashwani Kharola ${ }^{1}$, Dr SB Singh ${ }^{2}$ \\ Govt. Of India, Ministry of Defence \\ Institute of Technology Management (ITM), Defence R\&D Organisation \\ Mussoorie, Uttarakhand, India.
}

\begin{abstract}
This paper shows a elaborates new methodology for calculating Adhesive Strength of thin film coating using approximate fuzzy reasoning. This approach mainly focuses on nano and micro thin films for evaluating adhesion strength. The influence of various coating parameters(Critical force, loading rate, Displacement rate, Coefficient of friction, Tip radius and Tip wear) on quality of adhesion strength is considered. is used Modeling and Simulation is done with the help of fuzzy logic toolbox and results are shown.
\end{abstract}

Keywords: Fuzzy logic, coating parameters, Modeling, Simulation.

\section{INTRODUCTION}

The main purpose of the thin film coating application is to improve the surface properties of the materials while maintaining its bulk properties. The two main challenging issues that needed to be encountered in the coating process are cost and optimisation [1]. The adhesion strength is defined as the work done to separate a thin film from the substrate. Adhesive strength is very sensitive to measurement and evaluation conditions, such as strain rate, roughness, thickness, etc [2]. Scratch test is one of the most common method employed for measuring adhesion strength of a coating. It involves pulling a stylus [5] (mostly diamond) of spherical shape (200 m radius) against coating surface $[3,4]$. Some of the commonly used Scratch test are Progressive loading scratch test mode (PLST), constant load scratch test mode and multi-pass scratch test [6]. Recently non-destructive evaluation (NDE) have become ubiquitous in manufacturing systems because the technology is an inexpensive and reliable method to measure product requirements[7].

\section{FUZZY REASONING APPROACH FOR THE EVALUATION OF ADHESION STRENGTH OF THE NANO AND MICRO FILMS}

Fuzzy logic reasoning approach can be used as an effective tool for determining the Adhesion Strength of the nano and micro films. The existing International standards and knowledge of adhesion can be used as models/patterns for setting the fuzzy expert system, which could be used to identify the quality of practical adhesion strength. Also, artificial intelligence methods implemented in Matlab toolboxes [8] can be used in the 
process. The use of fuzzy logic, as a kind of "soft" computing techniques, enables us to overcome the problems encountered using "hard" computing techniques [9]. The advantage of using fuzzy logic reasoning is the application of linguistic information (linguistic variables) which is the basis for human communication [10]. Fuzzy logic controller determines the behaviour of a system by varying an input or output to that system with the help of fuzzy control rules [11]

\subsection{Designing of fuzzy reasoning model}

The fuzzy expert system uses a fuzzy if-then rule base consisting of a set of intuitive fuzzy rules, interpreting an input and producing a crisp output. In this study output (quality of adhesion) is significantly influenced by the input variables, which are: critical force, coating thickness, loading rate, displacement rate, substrate roughness, coefficient of friction, tip radius, tip wear and tip damage. Figure 1.0 shows the structure of Fuzzy Inference System (FIS) created in Matlab using Fuzzy Logic Toolbox [12]

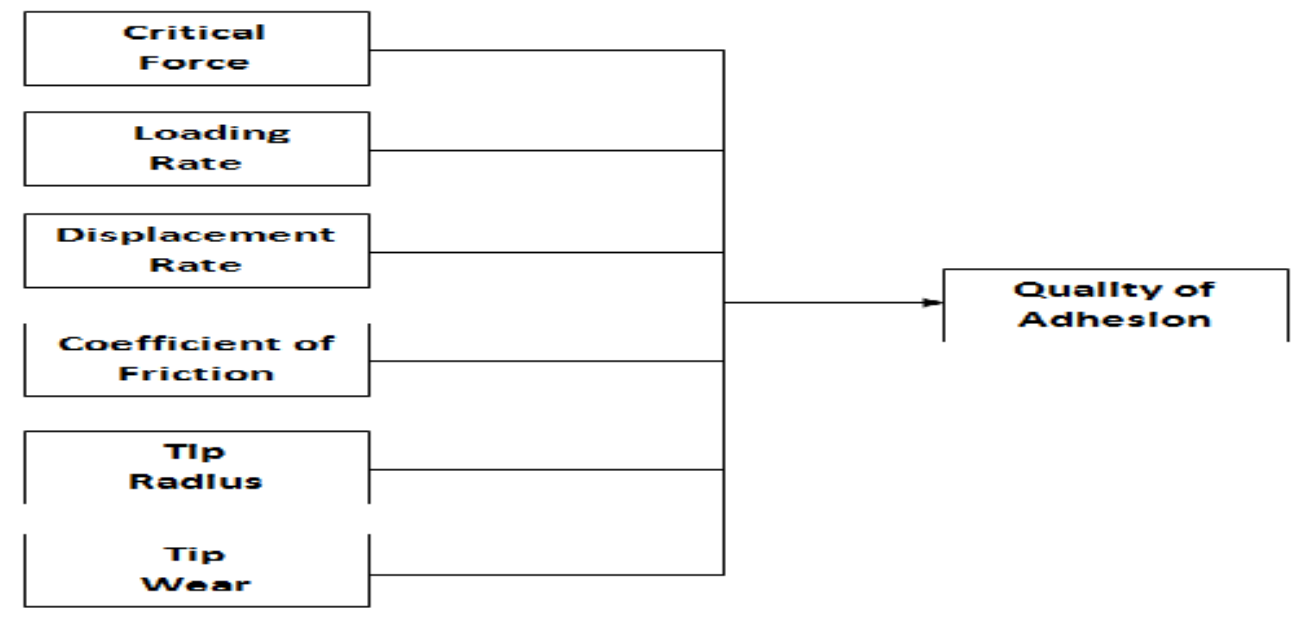

Figure 1.0 Structure of Fuzzy Inference System (FIS)

If we look at the figure 1.0 then we can see that inputs are combined to build Fuzzy Inference System (FIS) analysis. Structure of Fuzzy Inference System (FIS) in Fuzzy logic toolbox is shown in Figure 1.1

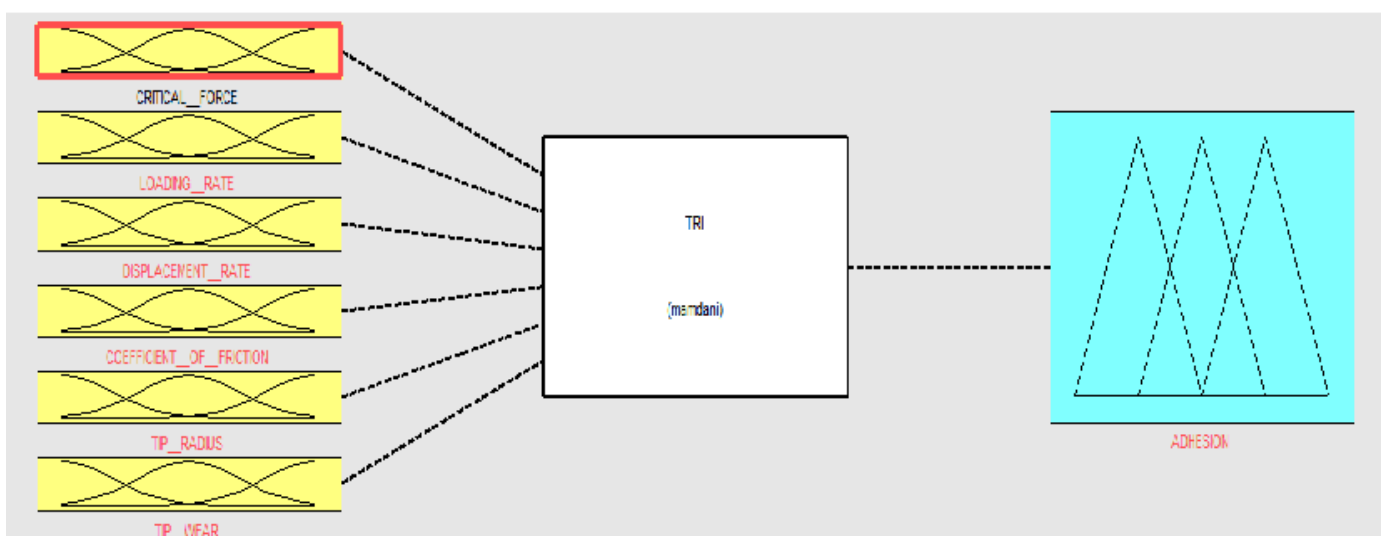

Figure 1.1 Structure of Fuzzy Inference System (FIS) in Fuzzy logic toolbox 
International Journal of Advances in Materials Science and Engineering (IJAMSE) Vol.3, No.3/4,October 2014

\subsection{Designing of Membership function's (MF's)}

Fuzzification of the constraints i.e. Input/Output with four linguistic variables using triangular MF's. The MF's for each of the constraint along with UOD are shown in figure 1.1 to figure 1.8

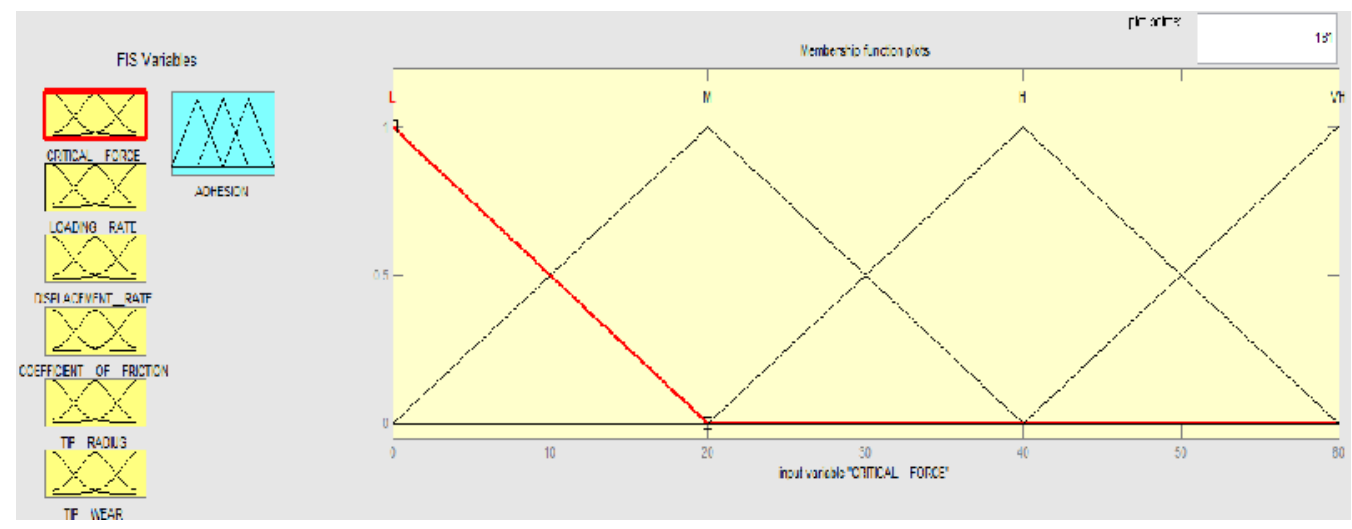

Figure 1.2 Fuzzified Input parameter 'Critical Force (Newton)'

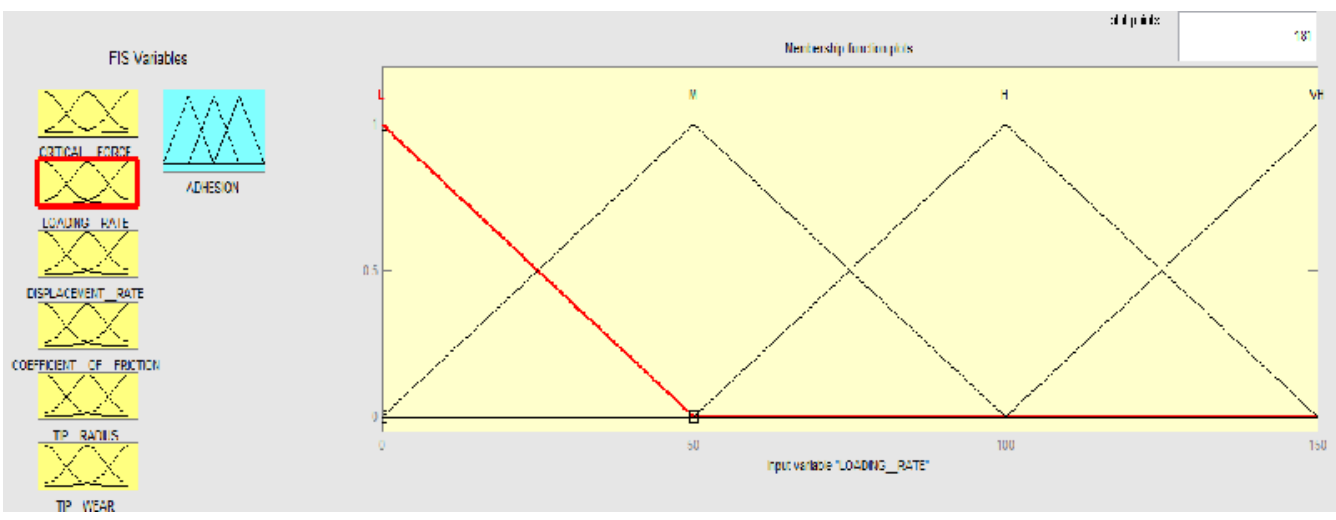

Figure 1.3 Fuzzified Input parameter 'Loading Rate(Newton/min)'
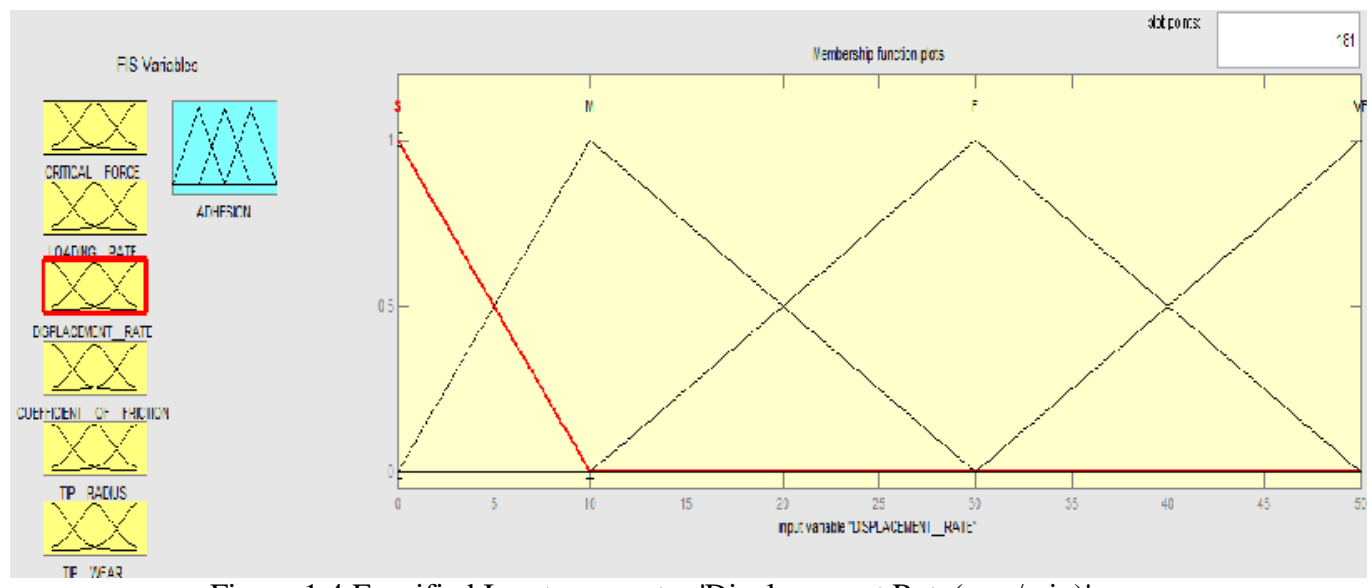

Figure 1.4 Fuzzified Input parameter 'Displacement Rate(mm/min)' 
International Journal of Advances in Materials Science and Engineering (IJAMSE) Vol.3, No.3/4,October 2014

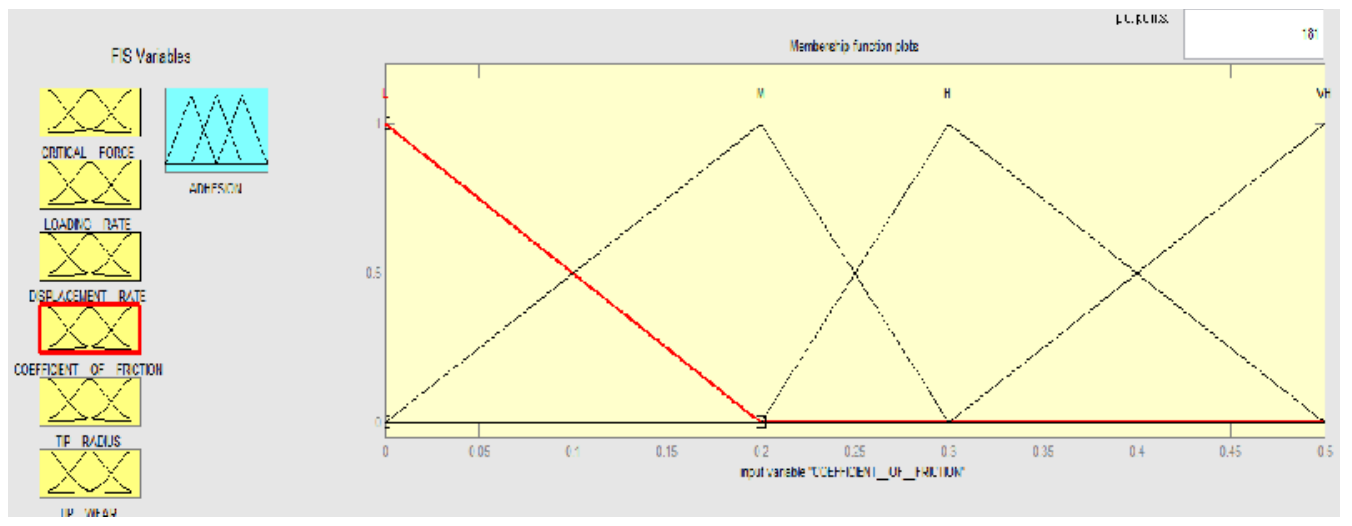

Figure 1.5 Fuzzified Input parameter 'Coefficient of Friction $(\mu)^{\prime}$

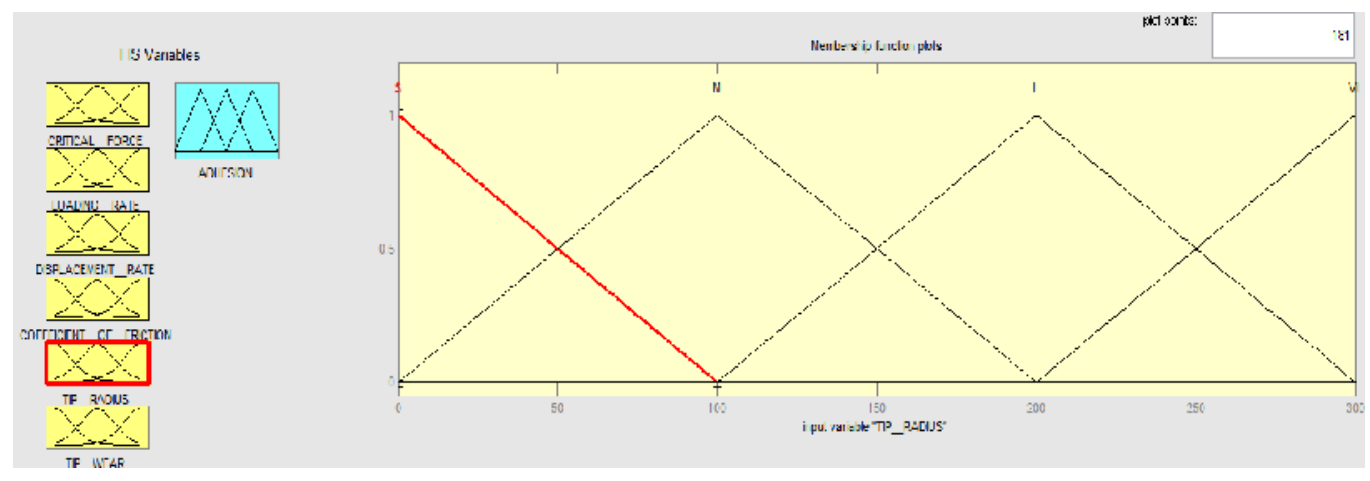

Figure 1.6 Fuzzified Input parameter 'Tip Radius(mm)'

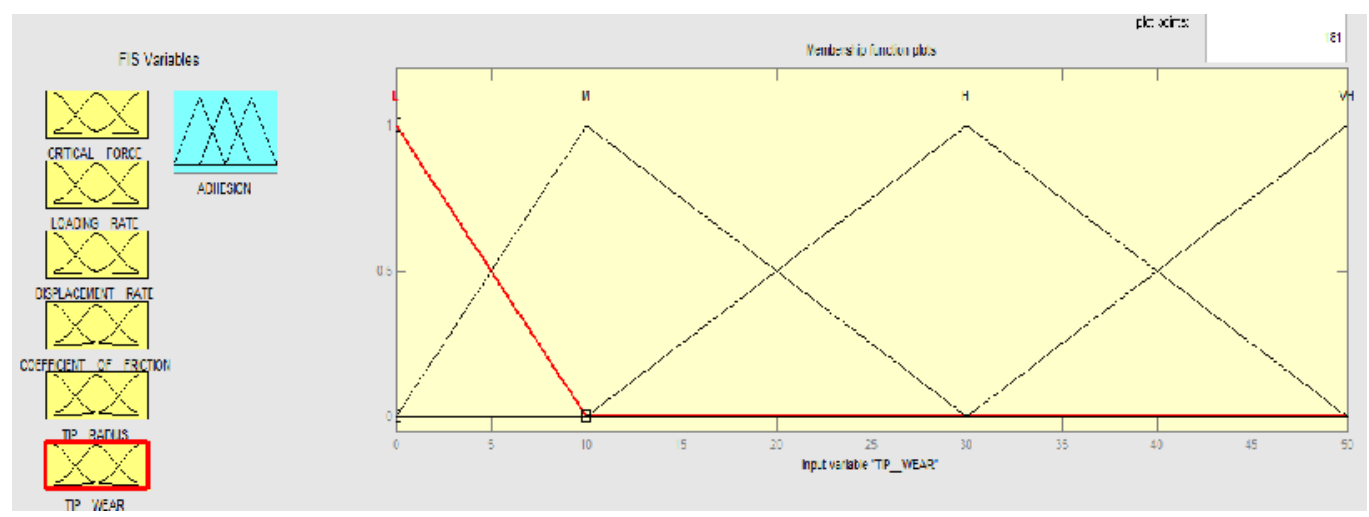

Figure 1.7 Fuzzified Input parameter 'Tip Wear $\left(\mathrm{mm}^{\wedge} 3 / \mathrm{min}\right)^{\prime}$ 


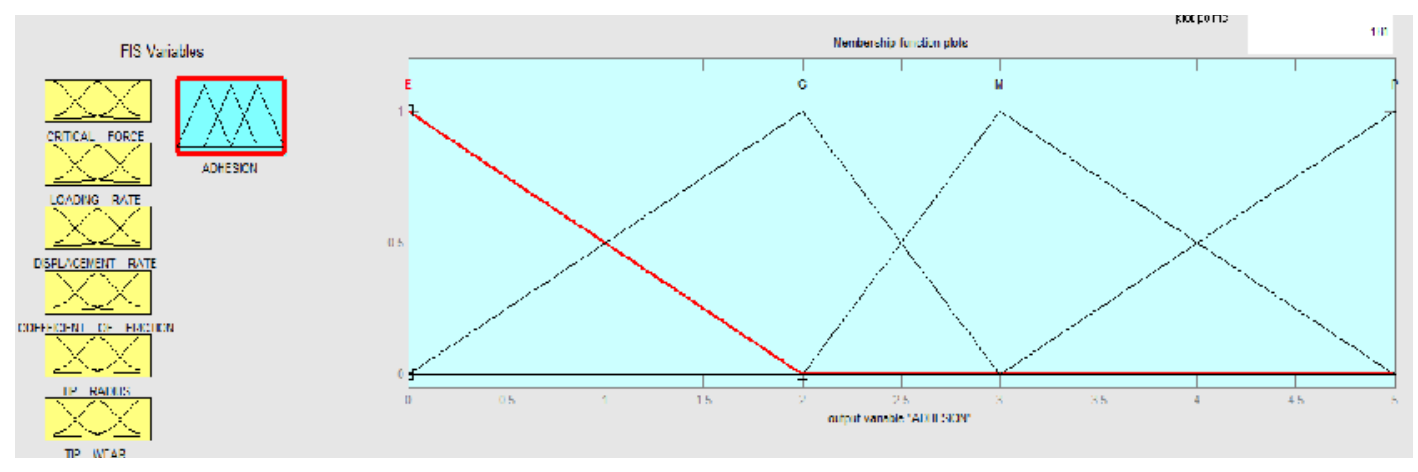

Figure 1.8 Fuzzified Output parameter 'Adhesion Strength $(\mathrm{KN} / \mathrm{m})^{\prime}$

\subsection{Designing of Fuzzy rules for controller}

This paper utilises fuzzy ordinal approach [13] to design if then fuzzy rules[14]. This approach has significantly reduced the number of rules. Each of the rules is given different degree of support (DOS) which determines the relative importance of each rule[15]. The fuzzy control rules along with DOS for the controller are given below:

(1) If 'Critical Force' is 'Low' then 'Adhesion' is 'Poor' (1)

(2) If 'Critical Force' is 'Medium' then 'Adhesion' is 'Moderate' (1)

(3) If 'Critical Force' is 'High' then 'Adhesion' is 'Good' (1)

(4) If 'Critical Force' is 'Very High' then 'Adhesion' is 'Excellent'(1)

(5) if 'Loading Rate' is 'Low' then 'Adhesion' is 'Excellent'(0.2)

(6) if 'Loading Rate' is 'Medium' then 'Adhesion' is 'Good'(0.2)

(7) if 'Loading Rate' is 'High' then 'Adhesion' is 'Moderate'(0.2)

(8) if 'Loading Rate' is 'Very High' then 'Adhesion' is 'Poor'(0.2)

(9) if 'Displacement Rate' is 'Small' then 'Adhesion' is 'Poor' (0.2)

(10) if 'Displacement Rate' is 'Medium' then 'Adhesion' is 'Moderate'(0.2)

(11) if 'Displacement Rate' is 'Fast' then 'Adhesion' is 'Good' (0.2)

(12) if 'Displacement Rate' is 'Very Fast' then 'Adhesion' is 'Excellent' (0.2)

(13) if 'Tip Radius' is 'Small' then 'Adhesion' is 'Excellent'(0.3)

(14) if 'Tip Radius' is 'Medium' then 'Adhesion' is 'Good'(0.3)

(15) if 'Tip Radius' is 'Large' then 'Adhesion' is 'Moderate'(0.3)

(16) if 'Tip Radius' is 'Very Large' then 'Adhesion' is 'Poor'(0.3)

(17) if 'Tip Wear' is 'Low' then 'Adhesion' is 'Excellent'(0.4)

(18) if 'Tip Wear' is 'Medium' then 'Adhesion' is 'Good'(0.4)

(19) if 'Tip Wear' is 'High' then 'Adhesion' is 'Moderate'(0.4)

(20) if 'Tip Wear' is 'Very High' then 'Adhesion' is 'Poor'(0.4)

As can be seen from above there are total 20 rules. As an example consider rule number 6 . In this rule if 'Loading Rate' is 'Medium' then 'Adhesion' is 'Good' with DOS equal to 0.2. A Surface Viewer for FIS is shown in Figure 1.9 which demonstrates the relationship between the input variables Critical force, loading rate and output variable Adhesion strength. 
International Journal of Advances in Materials Science and Engineering (IJAMSE) Vol.3, No.3/4,October 2014

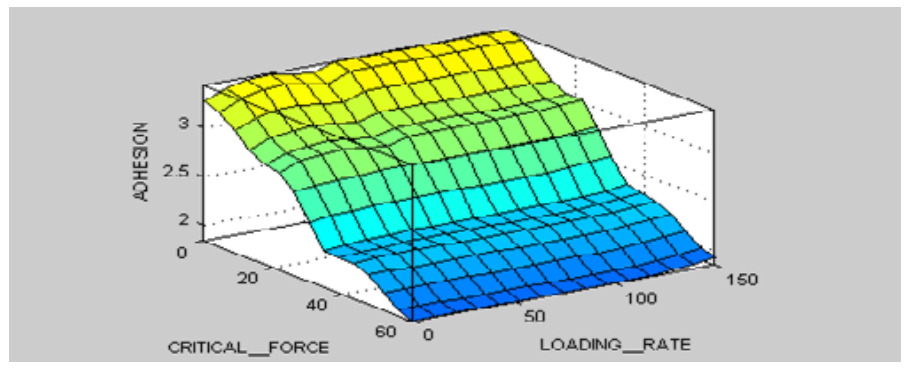

Figure 1.9 A Surface Viewer for FIS

As can be seen from the figure 1.9 if we have numerical value of Critical force $(\mathrm{N})$ and Loading rate $(\mathrm{N} / \mathrm{mm})$ in the scale of $0-60$ and $0-150$ respectively, then we can get the value of Adhesive strength $(\mathrm{KN} / \mathrm{m})$ in the range of $0-5$.

\section{SIMULATION RESULTS}

Results of simulations for different set of Inputs i.e. critical force, Loading rate, Displacement rate, Coefficient of friction, Tip radius and Tip wear are shown with the help of Tables and figures below:

\section{Set 1:}

\begin{tabular}{|c|c|c|c|}
\hline S.No & Inputs & Symbol & Value \\
\hline $\mathbf{1}$ & Critical force & a & 20 \\
\hline 2 & Loading rate & b & $\mathbf{5 0}$ \\
\hline 3 & Displacement rate & c & 10 \\
\hline 4 & Coefficient of friction & d & $\mathbf{0 . 2}$ \\
\hline 5 & Tip radius & e & 100 \\
\hline 6 & Tip wear & f & $\mathbf{2 5}$ \\
\hline
\end{tabular}

Table 1.0 Values of various Inputs

As can be seen from figure 2.0, for the inputs considered from Set 1, the value of the output i.e. Adhesive strength comes out to be $2.762 \mathrm{KN} / \mathrm{m}$.

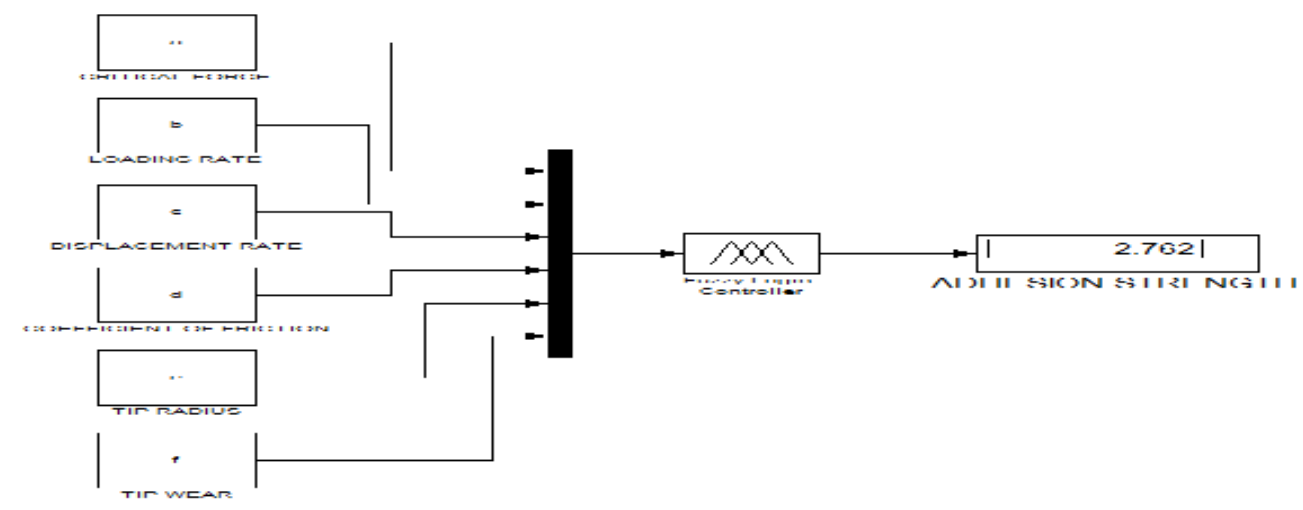

Figure 2.0 Simulation results 
Set 2:

\begin{tabular}{|c|c|c|c|}
\hline S.No & Inputs & Symbol & Value \\
\hline $\mathbf{1}$ & Critical force & a & $\mathbf{5 0}$ \\
\hline $\mathbf{2}$ & Loading rate & b & $\mathbf{1 0 0}$ \\
\hline $\mathbf{3}$ & Displacement rate & c & $\mathbf{3 0}$ \\
\hline $\mathbf{4}$ & Coefficient of friction & d & $\mathbf{0 . 3}$ \\
\hline $\mathbf{5}$ & Tip radius & e & $\mathbf{2 0 0}$ \\
\hline $\mathbf{6}$ & Tip wear & f & $\mathbf{5 0}$ \\
\hline
\end{tabular}

Table 1.1 Values of various Inputs

As can be seen from figure 2.1, for the inputs considered from Set 1, the value of the output i.e. Adhesive strength comes out to be $2.321 \mathrm{KN} / \mathrm{m}$.

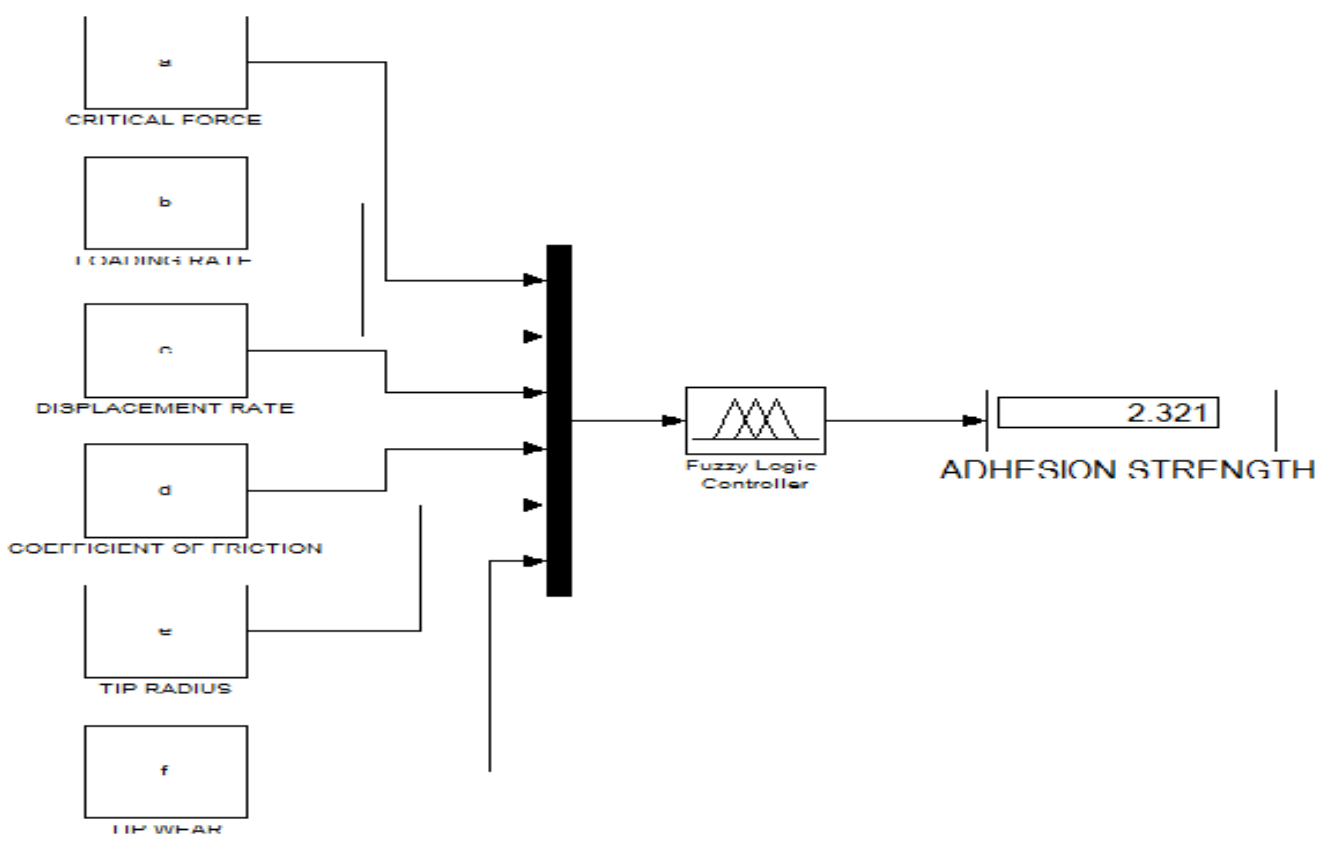

Figure 2.1 Simulation results

\section{CONCLUSION}

In this study a new approach for evaluation of Adhesive Strength of nano and micro thin films using fuzzy logic reasoning has been proposed. This methodology can be considered as an important non-destructive testing technique for evaluation of coating strength. The fuzzy reasoning methodology provides a more logical approach as compared to other conventional techniques. In this study we have incorporated fuzzy ordinal approach which proves to be an effective technique by reducing the number of fuzzy If-then rules. A Matlab Simulink model has been developed and the proposed framework optimises the related constraints. As a extension for future work one can use other types of MF's like Trapezoidal, Gaussian etc for better results. We can also consider other constraints which effects the adhesive strengths of thin-film coatings. 


\section{REFERENCES}

[1] Abdul Syukor Mohamad Jaya, Abd Samad Hasan Basari, Siti Zaiton Mohd Hashim, Habibollah Haron, Muhd. Razali Muhamad and Md. Nizam Abd. Rahman, "Application of ANFIS in Predicting of TiAlN Coatings Hardness", Australian Journal of Basic and Applied Sciences, 5(9): 1647-1657, 2011,ISSN 1991-8178

[2] Vladimır Socha, Patrik Kutliek, Slavka Viteckova, "The evaluation of the practical adhesion strength of biocompatible thin films by fuzzy logic expert system and international standards", Journal of electrical engineering, vol. 64, NO. 6, 2013, 354-360

[3] Standard C1624 (C1624-05) Test Method for Adhesion Strength and Mechanical Failure Modes of Ceramic Coatings by Quantitative Single Point Scratch Testing. Conshohocken: ASTM International, (2005).

[4] Standard ASTM D7187, "Test Method for Measuring Mechanistic Aspects of Scratch/Mar Behaviour of Paint Coatings by a Nano scratching", Conshohocken: ASTM International, (2005).

[5] P.Steinmann, H.Hintermann, "Adhesion of TiC and Ti(C,N) Coatings on Steel", J. Vac. Sci. Technol. 3 No. 6 (1985), 2394-2400.

[6] J.Valli, "TiN Coating Adhesion Studies Using the Scratch Test Method", J. Vac. Sci. Technol. 3 No. 6 (1985), 2411-2414.

[7] Jay Tillay Johnson, "Defect and thickness inspection system for cast thin films using machine vision and full-field transmission densitometry", A Thesis Presented to The Academic Faculty, School of George W. Woodruff School of Mechanical Engineering

[8] S.Sivanandam, S.Sumathi, S.Deepa," Introduction to Fuzzy Logic Using MATLAB", Springer, Berlin, 2010.

[9] L.Zadeh: Fuzzy sets, Information Control 8 (1965), 338-353.

[10] V. K. Dwivedi, " fuzzy based decision making for selection of fluid film journal bearing, International Journal of Fuzzy Logic Systems (IJFLS) Vol.2, No.3, July 2012

[11] Nabil Ibrahim El Sawalhi, "Modelling the Parametric Construction Project Cost Estimate using Fuzzy Logic", International Journal of Emerging Technology and Advanced Engineering Website: (ISSN 2250-2459, Volume 2, Issue 4, April 2012).

[12] Fuzzy Logic Toolbox: User's Guide MathWorks, www.mathworks.com/help/pdf_doc/fuzzy/fuzzy.pdf

[13] Asunobu Naitoh, Takeshi Furuhash and Yose Uchikawa, "A Variable Ordinal Structure Model for Fuzzy Reasoning and its Application to Decision Problem of Working Order" 1991 IEEE

[14] Adnan Shaout and Jaldip Trivedi, "Performance Appraisal System Using a Multistage Fuzzy Architecture", International Journal of Computer and Information Technology (ISSN: 2279 - 0764) Volume 02- Issue 03, May 2013.

[15] Dr. SB Singh, Ashwani Kharola, "Development of fuzzy logic model for performance rating (PR) of employee's", AMO - Advanced Modelling and Optimization, Volume 15, Number 3, 2013 